\title{
Eigenfrequency of Hydraulic Systems of Loading Device
}

\author{
Martin Vašina ${ }^{1, a}$, Lumír Hružík ${ }^{1}$ and Adam Bureček ${ }^{1}$ \\ ${ }^{1}$ VŠB-Technical University of Ostrava, Faculty of Mechanical Engineering, Department of Hydromechanics and Hydraulic Equipment, 708 \\ 33 Ostrava, Czech Republic
}

\begin{abstract}
Eigenfrequency of hydraulic systems belongs to important dynamic quantities. If the excitation frequency of a given hydraulic system is equal to the system eigenfrequency, high-amplitude pressure and flow pulsations can arise. It has a negative influence on load of hydraulic elements, system tightness etc. For this reason it is necessary to eliminate the operation of the hydraulic system at its eigenfrequency. The paper deals with experimental determination of the system eigenfrequency in various operating modes of the investigated loading device.
\end{abstract}

\section{Introduction}

Hydraulic systems are applied in many areas at the present time. They are used as drives of heavy duty equipment (e.g. cranes, loaders and excavators) [1]. Furthermore hydraulic systems are characterized by relatively small dimensions in comparison with different drives. It is their big advantage in comparison to other types of drives.

There are different requirements for hydraulic systems, e.g. energy costingness, purchase costs, reliability, dynamic properties, safety, weight, dimensions and service life. It is necessary to consider the requirements during the design process of a given hydraulic system [2].

This paper is focused on dynamics of hydraulic systems. Dynamical properties of hydraulic systems are also characterized by the system eigenfrequency, which depends on many parameters. The eigenfrequency can be determined by means of experimental measurements, mathematical simulations or simplified mathematical formulas.

The objective of this work is to experimentally investigate the eigenfrequency of a hydraulic system of the WM 185 hydraulic manipulator. The eigenfrequency was obtained under different working conditions of the manipulator.

\section{Eigenfrequency of hydraulic systems}

The eigenfrequency of hydraulic systems belongs to significant dynamic quantities. If the excitation frequency of a given hydraulic system is consistent with its eigenfrequency, high-amplitude pressure and flow pulsations are generated in the system. These pulsations have a negative influence on the system operation (e.g. on system tightness and cyclic loading of hydraulic elements). It is possible to determine the eigenfrequency of hydraulic systems by means of mathematical simulations, mathematical formulas or experimental measurements.

Mathematical models of hydraulic systems are described by a system of algebraic and differential equations. These equations are subsequently solved by Laplace transformation [3] or by computer programs (e.g. using Matlab, Mathematica or Mathcad software).

Simplified mathematical formulas [4] are used to an approximate determination of the system eigenfrequency, e.g. in the design phase. For example, the eigenfrequency $f_{0}$ of a rotary hydraulic motor is given by the formula:

$$
f_{0}=\frac{1}{2 \cdot \pi} \cdot \sqrt{\frac{k_{\varphi}}{J_{M}}},
$$

where $k_{\varphi}$ static stiffness of the rotary hydraulic motor, $\mathrm{N} \mathrm{m} ; J_{M}$ imoment of inertia of rotating masses, $\mathrm{kg} \mathrm{m}^{2}$. Similarly, the eigenfrequency $f_{0}$ of a linear hydraulic motor is given by the equation:

$$
f_{0}=\frac{1}{2 \cdot \pi} \cdot \sqrt{\frac{k_{h}}{m_{r e d}}}
$$

where $k_{h}$ static stiffness of the linear hydraulic motor, $\mathrm{N} \mathrm{m}^{-1} ; m_{\text {red }}$ reduced mass of moving parts, $\mathrm{kg}$. In the case of the linear hydraulic motor with a single piston rod and with minimum pressure (i.e. $p_{2} \rightarrow 0$ ) at its output (see figure 1 ), it is possible to express the eigenfrequency $f_{0}$ as follows:

$$
f_{0}=\frac{s_{1}}{2 \cdot \pi} \cdot \sqrt{\frac{K}{m_{\text {red }} \cdot\left(s_{1} \cdot x+V_{1}\right)}},
$$

\footnotetext{
${ }^{a}$ Corresponding author: martin.vasina@vsb.cz
} 
where $S_{1}$ piston area on its input side with the input working pressure $p_{1}, \mathrm{~m}^{2} ; x$ piston extension $(x \in<0, h>)$, $\mathrm{m} ; h$ piston stroke, $\mathrm{m} ; V_{1}$ liquid volume in an inlet transmission channel (i.e. between the linear hydraulic motor and the control valve $\mathrm{CV}), \mathrm{m}^{3}$. As shown in figure $1, Q_{1}$ is input flow rate of pressure liquid into the motor, $Q_{2}$ is output flow rate of the pressure liquid from the motor and $v$ is speed of movement of the motor.

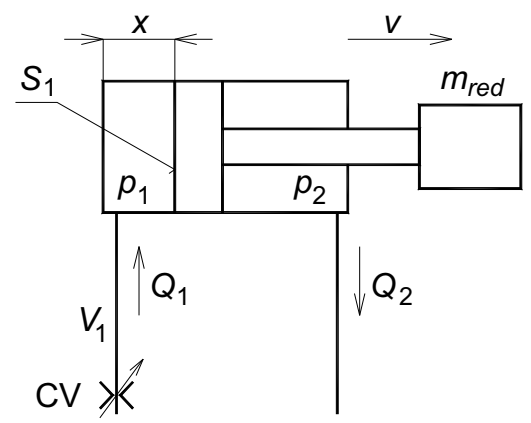

Figure 1. Schematic representation of linear hydraulic motor.

Experimental determination of the system eigenfrequency is realized by means of time-response characteristics, e.g. on basis of sudden changes of certain quantities. The time-response characteristics of a measured function $y(t)$ can be in general periodically damped or stochastic.

If the time dependence of the function $y(t)$ is periodically damped around the mean value $y_{m}$ (see figure 2), the system eigenfrequency is subsequently determined by the ratio $[5,6]$ :

$$
f_{0}=\frac{1}{T},
$$

where $T$ period of oscillation (see figure 2) of the function $y(t)$, s.

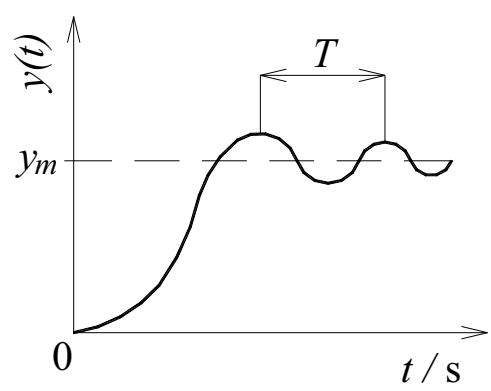

Figure 2. Example of time dependence of the periodically damped function $y(t)$.

In the case of the stochastic course (see figure 3) of the function $y(t)$, the system eigenfrequency is determined by means of the power spectral density [7]. Then the system eigenfrequency is obtained at the maximum value of the power spectral density [8].

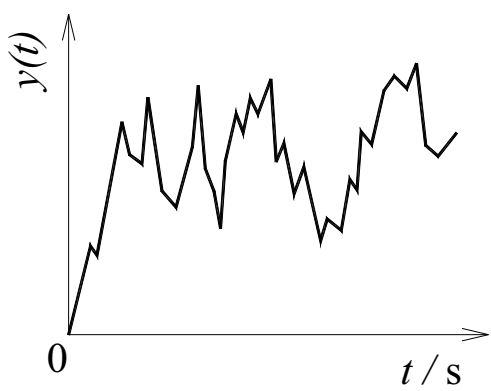

Figure 3. Example of stochastic course of the function $y(t)$.

\section{Investigated hydraulic system}

The system eigenfrequency was experimentally measured on the WM 185 hydraulic manipulator (see figure 4). The manipulator is used to loading and unloading of timber and other materials. The manually controlled WM 185 hydraulic manipulator is lifting equipment with the maximum lifting moment of $185 \mathrm{kN} \cdot \mathrm{m}$ and the maximum radius of $7.99 \mathrm{~m}$. The hydraulic manipulator consists of the following hydraulic motors [8]:

- Hydraulic motors of left and right stabilizing supports;

- Hydraulic motor for extension of supports;

- Turning hydraulic motor (for rotation of swivelling post);

- Hydraulic lifting motor (HLM);

- Two tilting hydraulic cylinders in order to achieve a pendulous motion of a telescopic boom;

- Hydraulic motor of the telescopic boom (HTM);

- Hydraulic motor of pliers (for opening or closing of the pliers) and

- Rotator (for turning the pliers).

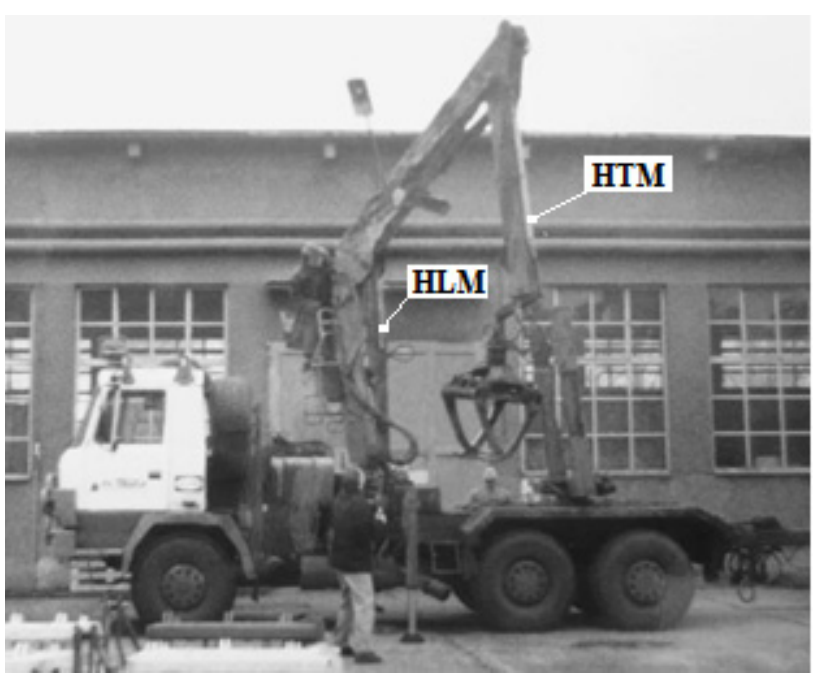

Figure 4. View of the investigated WM 185 hydraulic manipulator.

The hydraulic motors of both stabilizing supports and the hydraulic motor for extension of supports are used to ensure lateral stability of the manipulator. The other hydraulic motors are used for material handling (i.e. for 
lifting, lowering, loading and transloading of different materials).

The investigated hydraulic system belongs to closed center load sensing systems and is characterized by higher energy savings. The hydraulic system also consists of a hydraulic pump that is a pressure energy source of the system. The working principle of the WM 185 hydraulic manipulator and the schematic diagram of the hydraulic system are described in detail in [8].

\section{Experimental determination of system eigenfrequency}

The eigenfrequency of the investigated WM 185 hydraulic manipulator was experimentally measured under different working conditions, which were obtained on the basis of transient changes in behaviour of the hydraulic lifting motor (i.e. at a sudden stop of the lifting motor HLM in a given piston position of the motor). The other hydraulic motors did not move. They were in given positions at the same time. Furthermore the hydraulic motor of the telescopic boom HTM was located at several piston positions of this boom and the manipulator was loaded with different reduced masses during the measurements. The experimental measurement of the system eigenfrequency was purposely chosen for the lifting motor HLM due to the highest reduced masses $m_{\text {red }}$ of the lifting motor compared to the other hydraulic motors (see figure 4). As indicated in the Eq. (3), the reduced mass significantly reduces the system eigenfrequency $f_{0}$. For this reason the system eigenfrequency was determined under these working conditions of the hydraulic manipulator.

The time-response characteristics of the input pressure $p_{1}$ and the output pressure $p_{2}$ (see figure 1) of the lifting motor HLM were measured by means of the M5000 Hydrotechnik measuring equipment. The connection of this equipment on the WM 185 hydraulic manipulator is shown in figure 5 .

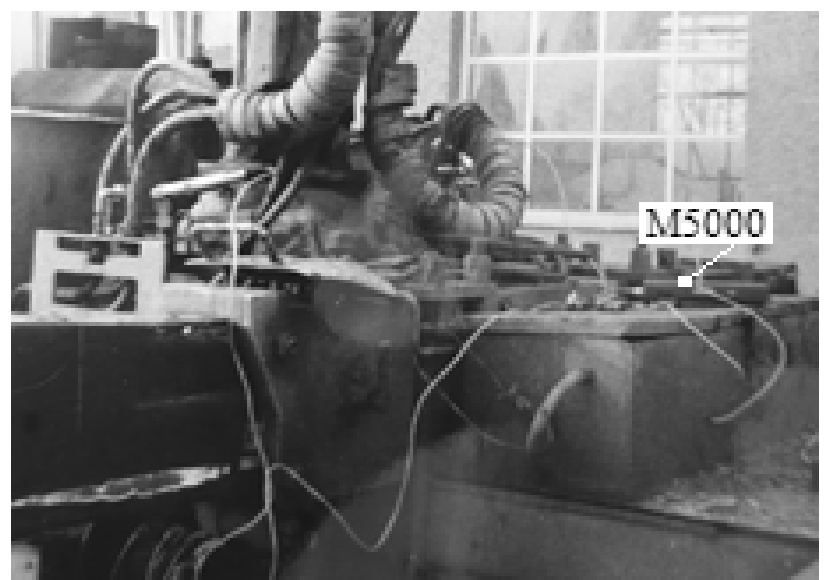

Figure 5. Connection of the M5000 Hydrotechnik measuring equipment

The time-response characteristic (see figure 6) of the investigated hydraulic manipulator was firstly measured during a sudden stop of the hydraulic lifting motor HLM (see figure 4 ) in the middle of its stroke (i.e. in the motor position $x=h / 2$ ). At the same time, the telescopic boom HTM with the piston position $x=0$ was unloaded. It is evident that the input pressure $p_{1}$ is subsequently changed during the time-response characteristic. The time dependence of the input pressure is periodically damped around the mean value $p_{1} \cong 64 \cdot 10^{5} \mathrm{~Pa}$ with the period of oscillation $T \cong 0.55 \mathrm{~s}$. Then it is possible to determine the system eigenfrequency from the Eq. (4):

$$
f_{0}=\frac{1}{T}=\frac{1}{0.55}=1.82 \mathrm{~Hz}
$$

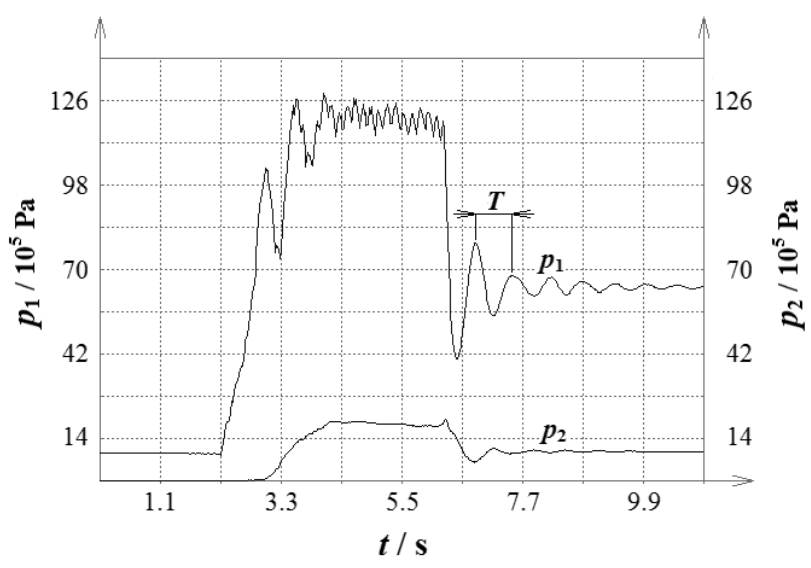

Figure 6. Time dependencies of the pressures $p_{1}$ and $p_{2}[8]$.

The system eigenfrequency was subsequently determined during a sudden stop of the hydraulic lifting motor HLM in the position $x=2 \mathrm{~h} / 3$. In this case the telescopic boom HTM with the piston position $x=0$ was loaded. It is visible (see figure 7) that the input pressure $p_{1}$ is periodically damped with the period of oscillation $T \cong 1.17 \mathrm{~s}$. For this reason the system eigenfrequency is determined as the reciprocal of the period:

$$
f_{0}=\frac{1}{T}=\frac{1}{1.17}=0.85 \mathrm{~Hz} .
$$

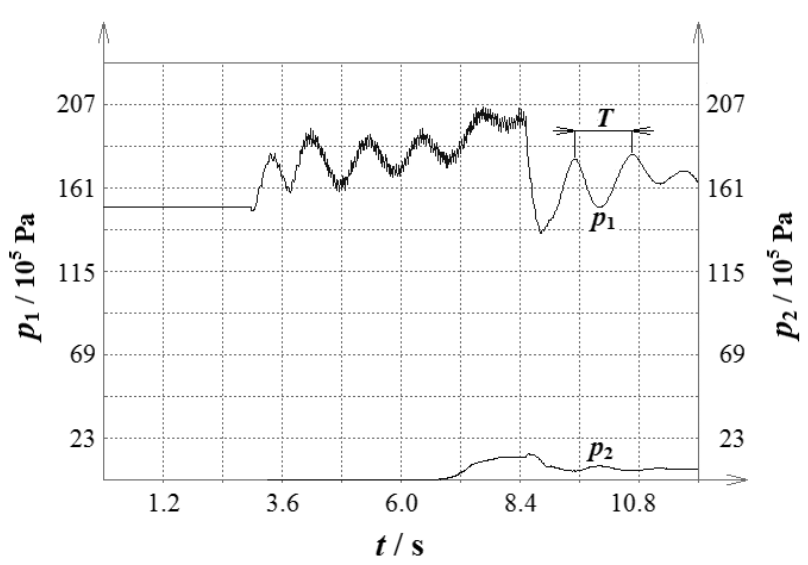

Figure 7. Time dependencies of the pressures $p_{1}$ and $p_{2}$ [8].

Figure 8 demonstrates the time dependencies of the pressures $p_{1}$ and $p_{2}$ during a sudden stop of the hydraulic lifting motor HLM in the position $x=2 \mathrm{~h} / 3$. At the same time the telescopic boom HTM with the piston position $x=h$ was again loaded. It is obvious that the input pressure $p_{1}$ oscillates around the mean value 
$p_{1} \cong 210 \cdot 10^{5} \mathrm{~Pa}$ with the period of oscillation $T \cong 1.34 \mathrm{~s}$. Then, the eigenfrequency of the investigated hydraulic manipulator is determined as follows:

$$
f_{0}=\frac{1}{T}=\frac{1}{1.34}=0.75 \mathrm{~Hz}
$$

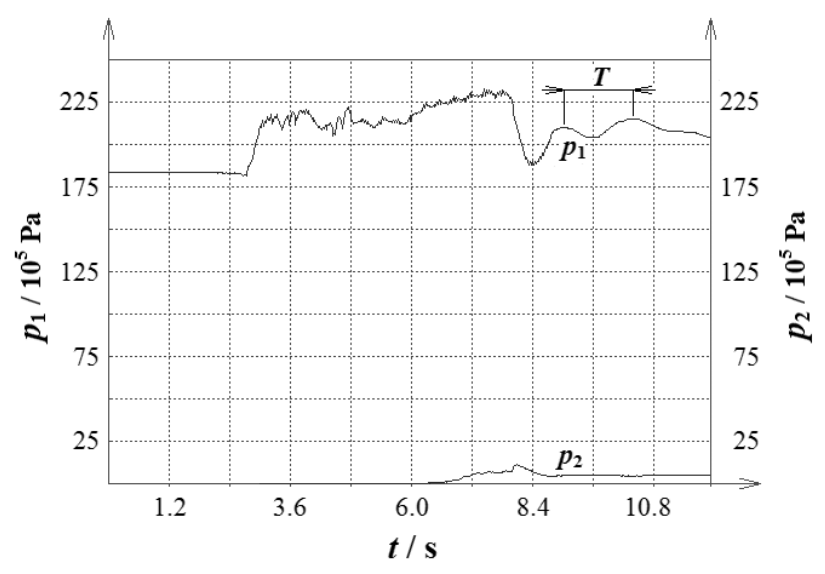

Figure 8. Time dependencies of the pressures $p_{1}$ and $p_{2}[8]$.

It is evident from the above-mentioned time-response characteristics (see figures 6-8) that the eigenfrequency of the investigated WM 185 hydraulic manipulator is very low under the given working conditions. Nevertheless, it is recommended to ensure a minimum value of the system eigenfrequency $f_{0 \text { min }}$ during operation of the hydraulic system. In the case of the investigated hydraulic manipulator, which is represented as a load compensation system [4], the minimum eigenfrequency $f_{0 \min }=4 \mathrm{~Hz}$. It is evident that this frequency criterion is not fulfilled. This fact can lead to many complications during operation of the hydraulic system. It can be reflected in increased load of hydraulic elements, decreased system tightness and excessive noise and vibration. At worst, low values of the eigenfrequency can also lead to an accident of the system. It is also evident from the experimental measurements that the system eigenfrequency decreases with increasing piston extension $x$ of the motors HLM and HTM and with increasing reduced mass of moving parts $m_{\text {red }}$. It is in accordance with the Eq. (3).

It can be concluded that low values of the system eigenfrequency were probably caused by system aeration. Air bubbles in liquids have a negative influence on the system stiffness and dynamic behaviour of the investigated hydraulic manipulator. For this reason it is necessary to properly deaerate the hydraulic system. It can be also caused by a relatively large reduced mass $m_{\text {red }}$ of moving parts. Furthermore, it is possible to perform suitable precautions when designing the system. According to the Eq. (3), it is suitable to ensure the minimum liquid volume $V_{1}$ in the applied hydraulic system and to increase the piston area $S_{1}$ in the design phase.

\section{Conclusions}

The purpose of this paper was to experimentally determine the eigenfrequency of the WM 185 hydraulic manipulator that is used to loading and unloading of timber and other materials. The eigenfrequency was determined under different working conditions from time-response characteristics.

It was found in this study that the experimentally determined values of the system eigenfrequency were very low. It can lead to different failures during operation of the hydraulic manipulator. For this reason it is necessary to increase the system eigenfrequency through appropriate measures. It is suitable to properly deaerate the hydraulic system in order to increase its stiffness. Furthermore it is possible to reduce the reduced mass of moving parts and to perform different precautions when designing the system.

It is also desirable that the excitation frequency of a hydraulic pump in a given hydraulic system did not correspond to the system eigenfrequency under given working conditions. If the excitation frequency and the system eigenfrequency are consistent, it can have a negative influence on dynamic behaviour of the hydraulic system.

\section{References}

1. M. Ochiai, S. Ryu, 7th JFPS 2008, Hybrid in construction machinery, (Toyama, 2008)

2. P. Drexler, H. Faatz, F. Feith, H. Geis, J. Morlok, E. Wiesmann, The Hydraulic Trainer Volume 3 Planning and Design of Hydraulic Power Systems (Mannesmann Rexroth GmbH, Lohr am Main, 1988)

3. A. Furman, S. P. Neumam, Advances in Water Resources 26, 12 (2003)

4. H. Dörr, R. Ewald, J. Hutter, D. Kretz, F. Liedhegener, A. Schmitt, The Hydraulic Trainer Volume 2 - Proportional and Servo Valve Technology (Mannesmann Rexroth GmbH, Lohr am Main, 1986)

5. M. S. Chandrasekar, M. Pushpavanam, Electrochimica Acta 53, 8 (2008)

6. M. D. Bootman, M. J. Berridge, P. Lipp Cell 91, 3 (1997)

7. P. Laguna, G. B. Moody, R. G. Mark, IEEE Transactions on Biomedical Engineering 45, 6 (1998)

8. M. Vašina, Energy saving hydraulic systems of lifting and loading equipment mounted on lorries (VŠB - Technical University of Ostrava, Czech Republic, 2000)

\section{Acknowledgements}

The work presented in this paper was supported by a grant SGS "Investigation of dynamics of fluid systems" SP2015/95. 